\title{
Handgrip Strength and Ultrasonographically-measured Lower Arm Muscle Thickness in Hospitalised Older Adults: The SARCopenia and Ultrasound $3^{\text {rd }}$ Pilot Study
}

\author{
(1) Thomas Acke1, (1) Anne-Marie De Cock1, (1) Vandewoude Maurits², (1) Stany Perkisas² \\ 1 University Center of Geriatrics, Antwerp, Belgium \\ 2 University Center of Geriatrics, Antwerp, Belgium and Belgian Ageing Muscle Society, Antwerp, Belgium
}

\begin{abstract}
Objective: The SARCopenia and UltraSound $3^{\text {rd }}$ (SARCUS3) pilot study aims to determine the relationship between ultrasound (US)-based lower arm muscle thickness and handgrip strength in hospitalised older adults.

Materials and Methods: SARCUS3 is a single-centre cross-sectional study (Ziekenhuis Netwerk Antwerpen, Campus Middelheim, Antwerp, Belgium). For inclusion, all patients admitted to a geriatric ward were screened. US was used to measure the thickness of the lower arm muscles. On the other hand, a Jamar dynamometer was used to measure handgrip strength.

Results: A total of 83 patients were included in the data analysis (48 women, 35 men, mean age 84 years). According to the Shapiro-Wilk test, the lower arm muscle thickness and square root of handgrip strength had a normal distribution. The scatterplot and line of best fit suggested that the two variables had a linear relationship. Pearson's correlation coefficient was $0.287(p=0.051)$ for women and $0.361(p=0.036)$ for men for the USmeasured muscle thickness of the lower arm and square root of handgrip strength. A linear regression analysis of the data from the participating men revealed that the best estimate for handgrip strength can be calculated using the formula: handgrip strength $(\mathrm{kg})=[2.773+0.061 \times$ lower arm muscle thickness $\left.\left(\mathrm{mm}^{2}\right)\right]$, with an adjusted $\mathrm{R}$ square of 0.103 .

Conclusion: This pilot study, using US-based muscle measurements, discovered a significant positive relationship for men and a borderline nonsignificant relationship for women between lower arm muscle thickness and handgrip strength. Furthermore, muscle thickness alone can explain up to 10.3\% of the measured variability of handgrip strength in men. To our knowledge, this study is the first to show that US-based measurements of the lower arm are related to handgrip strength in a group of hospitalised older people. More research is required to identify other factors that influence lower arm muscle strength in hospitalised older adults.
\end{abstract}

Keywords: Sarcopenia, grip strength, muscle thickness, ultrasound, older adults, age-related changes, frailty

\section{Introduction}

Sarcopenia was first described by Irwin Rosenberg in 1989 as a deficiency of muscle mass (1). During the following years, the terminology for sarcopenia changed according to the continuous increase in knowledge about the syndrome (2-4). In 2010 the European Working Group on Sarcopenia in Older People (EWGSOP) published a consensus definition and diagnostic criteria for age-related sarcopenia, with an update (EWGSOP2) in $2019(5,6)$. The EWGSOP2 definition states that sarcopenia is a progressive and generalized skeletal muscle disorder that is associated with an increased likelihood of adverse outcome including falls, fractures, physical disability and mortality (6). Low muscle strength is used as the main parameter of sarcopenia according the EWGSOP2 (6). Whenever low muscle strength is present, the term "probable sarcopenia" can be used according EWGSOP2 criteria (6). If there is additionally either low muscle quantity or quality present, the diagnose of sarcopenia is conformed (6). Low physical performance is furthermore used to grade the severity of sarcopenia (6).

Address for Correspondence: Thomas Acke, University Center of Geriatrics, Antwerp, Belgium

Phone: +32/494617206 E-mail: Thomas.acke@zna.be ORCID: orcid.org/0000-0002-8347-2454

Received: 19.11.2020 Accepted: 14.12.2020

Cite this article as: Acke T, De Cock AM, Maurits V, Perkisas S. Handgrip Strength and Ultrasonographically-measured Lower Arm Muscle Thickness in Hospitalised Older Adults: The SARCopenia and Ultrasound $3^{\text {rd }}$ Pilot Study. Eur J Geriatr Gerontol 2021;3(2):108-116

${ }^{\circ}$ Copyright 2021 by the Academic Geriatrics Society / European Journal of Geriatrics and Gerontology published by Galenos Publishing House. 
Defining the standard methods to measure muscle strength, quantity or quality remains subject of discussion (6). Handgrip strength was acknowledged by the EWGSOP2 as a convenient method to evaluate low muscle strength. The method is standardized and cut-off points for men and women were confirmed $(6,7)$. A measured handgrip strength $<27 \mathrm{~kg}$ for men and $<16 \mathrm{~kg}$ for women indicates the presence of low handgrip strength (6). Handgrip strength is a well-studied variable (8). Similar to sarcopenia, low handgrip strength has been associated with an increased likelihood of adverse outcome including falls (9), fractures (10), physical disability (11) and mortality $(12,13)$.

On the other hand, a suitable method to measure the muscle quality and muscle quantity in daily clinical practice is still undetermined. To date, computed tomography (CT) and magnetic resonance imaging (MRI) based measurements are regarded as the gold standard and dual-energy X-ray absorptiometry (DXA) as the preferred alternative for measuring muscle mass (6). However, these techniques are complex, expensive and unavailable in many centers making them of little use in daily clinical practice. Bioelectrical impedance analysis (BIA) is an additional, upcoming technique for measuring muscle mass $(6,14)$. Due to a lack of standardization and validation of the prediction equations in specific populations, its use in daily clinical practice remains rather limited $(6,14)$. Furthermore, BIAequations strongly depend on the patients fluid balance, which can greatly differ between healthy subjects and hospitalized older adults (15). The EWGSOP2 provides cut-off points for BIA and DXA - but not for CT or MRI-that indicate the presence of low muscle quantity (6). With an appendicular skeletal muscle mass (ASM) $<20 \mathrm{~kg}$ for men and $<15 \mathrm{~kg}$ for woman or an ASM/ height $^{2}<7.0 \mathrm{~kg} / \mathrm{m}^{2}$ for men and $<5.5 \mathrm{~kg} / \mathrm{m}^{2}$ conforming the presence of low muscle quantity $(6,16,17)$.

Despite a wide range of available technics and even recognized cut-off points for some of them, it remains unfeasible to measure muscle mass in daily clinical practice. An alternative for the aforementioned techniques however, might be found in ultrasonography $(6,18)$.

Ultrasound (US) is an inexpensive, portable, non-invasive technique without the need for ionizing radiation (18). Previous studies on US-based measurements of muscle quantity suggest a significant correlation with CT and MRI based measurements (19-21). In regard to the lower arm muscles, a recent study observed a strong correlation between the lower arm muscle thickness, measured as the distance between the subcutaneous adipose tissue-muscle interface and the muscle-bone interface of the ulnae/radius, and MRI-measured cross- sectional area of the flexor and extensor components of the lower arm (22). Multiple studies have shown a good intra- and inter-observer, test-retest reliability (23-26), and feasibility in assessing small muscle groups with the use of US (18,27-29).
Recommendations about the measurement of muscle quality in daily clinical remain scarce (6). As to date, mostly CT and MRI based measurement have been used in research to asses muscle quality (6). Similar to CT and MRI-based measurements, US can assess muscle quality directly via specific tissue characteristics $(18,30)$. Cadaver studies have shown that US is a valid tool for assessing basic architectural parameters $(31,32)$. As yet, there is no consensus as to which technique is to be used to provide qualitative information on muscles (6). The EWGSOP2 doesn't make any recommendations nor provides cut-off points for any qualitative muscle parameter (6). US-based measurements could play a major future role in the evaluation of sarcopenia as it combines muscle quality and quantity assessment without the downsides of CT, MRI, DXA or BIA (18).

Confirming the viability of use of this new US-based strategy could improve the diagnostic process of sarcopenia in regular practice. This includes describing the ideal detection area in older patients and gaining further insights in the relationship between muscle quality, quantity and muscle strength. Previous studies examining the muscle quantity and muscle strength relationship solely focused on healthy community dwelling older adults $(33,34)$. Therefore, the aim of this study is to question the relationship between US based measurements of lower arm muscle quantity and handgrip strength in hospitalized older adults.

\section{Materials and Methods}

\section{Study design}

The SARCopenia and UltraSound $3^{\text {rd }}$ study (SARCUS3) was a single centre cross-sectional study. This study was approved by our Local Hospital's Ethics Committee (approval no: 5226).

\section{Study population}

All patients admitted to the acute and orthogeriatric wards of the Ziekenhuis Netwerk Antwerpen campus Middelheim (Antwerp, Belgium) between the $1^{\text {st }}$ of May 2019 until the $31^{\text {th }}$ of December 2019 were eligible and screened for inclusion. Patient had to be over 65 years old and be able to comply with the study protocol to be included.

All patients with known cognitive problems (either mild cognitive impairment or dementia) or who were diagnosed with cognitive problems (either mild cognitive impairment, dementia or delirium) during hospitalization were excluded. Additionally, all patient with recent surgery or trauma ( $<3$ months) of the dominant arm, severe electrolyte disturbances, hyper- or hypovolemia or neuromuscular disease were excluded. A full list of the exclusion criteria is provided in the supplementary material. Written informed consent was obtained from all subjects. 


\section{Collection of descriptive data}

All information on the reason for admission, substance use, biometrical information, medication use and length of stay was either retrieved from the patient themselves or the patient's medical record. The Cumulative IIIness Rating Scale-Geriatric (CIRS-G) was used to asses co-morbidity. It rates 14 different organ systems (score 0 to 4), with a higher score implying a higher disease burden and with a maximum score of 56 (35). The mini nutritional assessment-short form (MNA-SF), a 6 item questionnaire, was used to identify patients who were are either at risk or had malnutrition (36). A score of 0 to 7 implying malnutrition, a score of 8 to 11 being at risk of malnutrition and a score of 12 to 14 meaning a normal nutritional status. The SARC-F score was used to screen for sarcopenia. The SARC-F rates 5 components (Strength, Assistance in walking, Rise from a chair, Climb stairs, Falls) and ranges from 0 to 10. A score of 0 meaning no characteristics of sarcopenia are present (37). In accordance with the EWGSOP2 criteria, probable sarcopenia was present when low handgrip strength $(<27 \mathrm{~kg}$ for men and $<16 \mathrm{~kg}$ for women) was confirmed (6). The FRAlL scale, a frailty screening stool ranging from 0 to 5 , was used to describe the presence of frailty in the study population $(38,39)$. A score of 3-5 indicating the presence of frailty, a score of 1-2 pre- frailty, and a score of 0 a health status.

\section{US based muscle measurements}

US based measurements of muscle thickness were used to quantify lower arm muscles. As these muscles are rather small, especially in older adults, measuring the thickness of an entire muscle compartment was chosen rather than measuring individual muscles. This approach deemed more straightforward than the more complex volume-based measurement of a single muscles. The dominant forearm muscles were measured while the participants were seated. The forearm was placed on a table to rest. The elbow was flexed to $120^{\circ}$ and the forearm put in
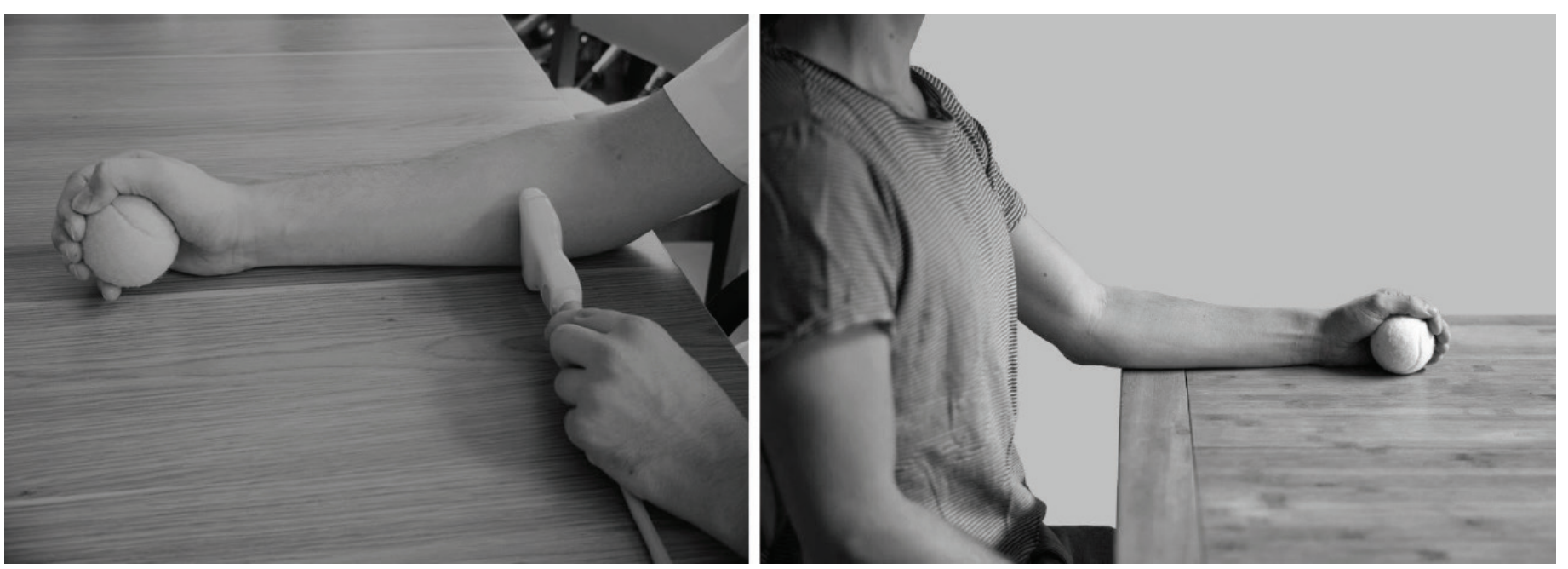

midprone (neutral position). The wrist was at $15-30^{\circ}$ of extension (dorsiflexion). Patients were asked to hold a Wilson US 4 tennis ball (Wilson Sporting Goods Company, United States of America) without squeezing it, to ensure correct positioning. A tennis ball was used as this is a standardized measure throughout the world. Muscle thickness was measured using brightness (B)-mode on an Aplio 300 (Canon Medical Systems Europe, the Netherlands). A 5 $\mathrm{cm}$ wide, 7.5 and $10 \mathrm{MHz}$ linear transducer with a scanning head coated with water-soluble transmission gel was used. Muscle thickness was measured at the proximal $1 / 3$ of the distance between the tip of the olecranon and the ulnar styloid process. An example of probe and patient positioning is shown in Figure 1. The probe was placed perpendicular on the medial side of the forearm. The distance between the subcutaneous adipose tissue-muscle interface and muscle-bone interface of the ulna was measured (Figure 2). All measurements were repeated three times and the mean value of these measurements was used. Muscle thickness was measured within 4 days of admission in order to minimize the risk of acute hospital admission related muscle wasting (40). Furthermore, in order to comply with the US study protocol patients had to be able to walk and sit up in a chair. Thus, reducing the risk of acute bed rest-induced muscle loss.

\section{Handgrip strength}

Handgrip strength was measured using a Jamar hydraulic hand dynamometer (Lafayette Instrument Company, United States of America), according to the American Society of Hand Therapists protocol (ASHT) $(41,42)$. Patients were seated in a chair without arm rests. There feet had to be on the ground. Hips had to be as far back in the chair as possible and the hips and knees positioned at approximately $90^{\circ}$. Shoulders had to be adducted and in a neutral position. The elbow had to be flexed at $90^{\circ}$ angle and the forearm was held in a midprone (neutral position). The wrist was held between 15 to $30^{\circ}$ of extension (dorsiflexion) and 0 to $15^{\circ}$ of ulnar deviation. The mean of three consecutive trials

Figure 1. Probe (left) and patient (right) positioning 
was used. A rest of at least 15 seconds was allowed between trials. Instructions to the patient were provided according to the ASHT-protocol.

\section{Statistics}

Statistical analysis was done by using Statistical Product and Service Solutions (SPSS) Statistics (International Business Machines Corporation, United States of America) version 26 software. Continuous variables are expressed as mean and standard deviation. Nominal variables are presented as percentages. Ordinal variables are presented as median and interquartile range. Handgrip strength and lower arm muscle thickness were checked for a normal distribution with a Shapiro-Wilk test. Data of muscle quantity and handgrip strength were plotted in a scatterplot and the correlation examined by calculation of the Pearson's correlation coefficient. The null hypothesis being that there is no relationship between muscle quantity and handgrip strength. The correlation was deemed significant at a 0.05 level (two tailed). In case of a significant correlation, a simple linear regression analysis will be performed. An additional bivariate analysis was performed using a correlation matrix. In case of multiple variables that

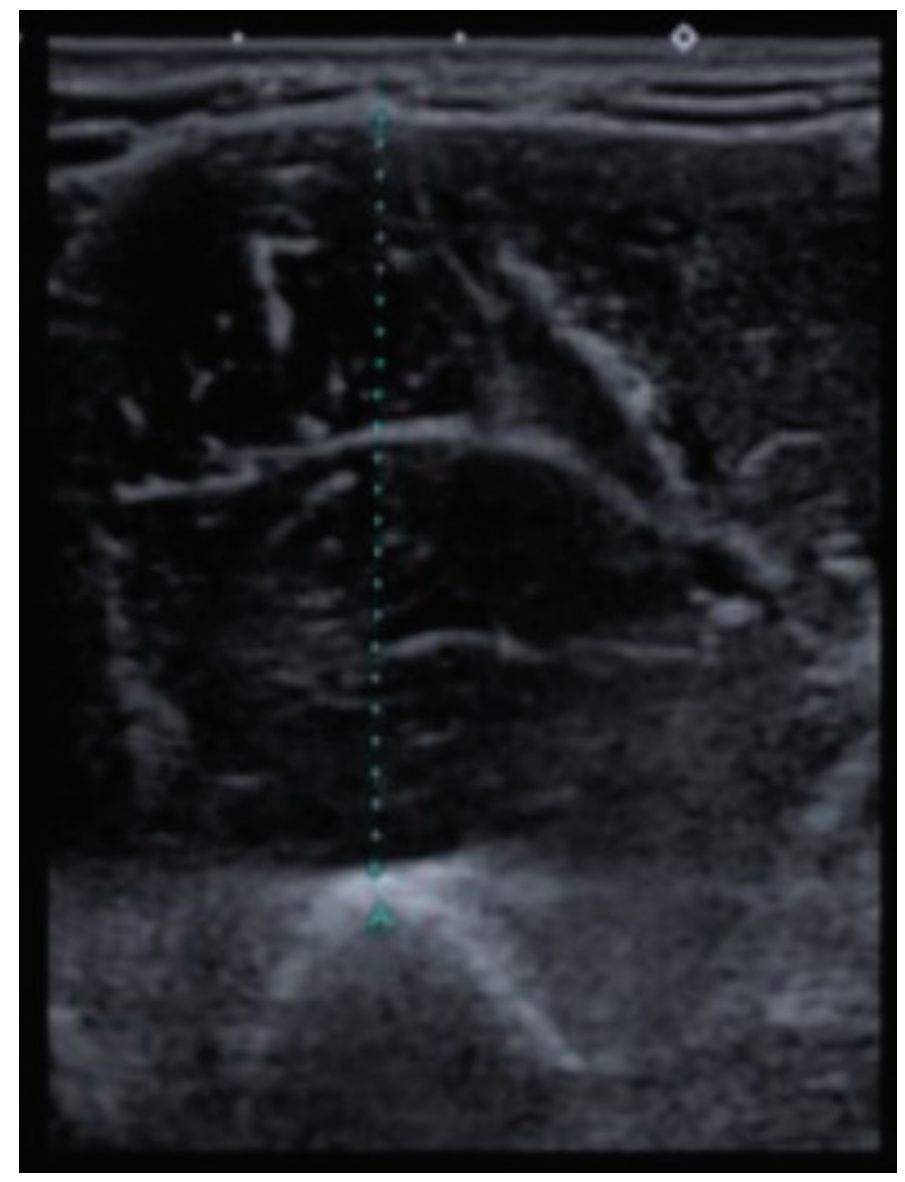

Figure 2. Muscle thickness. Dotted line: the distance between the subcutaneous adipose tissue- muscle interface (top) and muscle-bone interface of the ulna (bottom) correlated (Spearman's rho $>0.250, p>0.05$ ) to handgrip strength, a multiple regression analyze will be performed to further explore the relationship between lower arm muscle thickness and handgrip strength.

Before the start of the study a power analysis was performed using G*Power version 3.1.9.3 software (Heinrich HeineUniversiteit Düsseldorf, Germany) presuming there would be a weak correlation (0.3) between handgrip strength and lower arm muscle thickness. A required sample size of 82 patients was calculated.

\section{Results}

\section{Patient characteristics}

In total, 100 patients were included in the SARCUS3 study between the $1^{\text {st }}$ of May 2019 and the $30^{\text {th }}$ of November 2019. Seventeen patients had to be excluded: fifteen patients due to a new diagnose of either mild cognitive impairment or dementia, one patient because of a new diagnose of multiple sclerosis and one patient because of generalized edema caused by heart failure. After all exclusions, a total of 83 patients remained for data analysis. A summary of the patient characteristics is shown in Table 1. The mean age for women was $84.51 \pm 5.29$ years and for men $84.19 \pm 6.01$ years. Women represented $57.8 \%$ of the total population. On average, patients had serious comorbidities as is expressed by a high mean CIRS-G score of $10.08 \pm 4.20$ for men and $12.71 \pm 5.15$ for women. Patients where admitted for various reasons, mostly because of infections (20 patients), gait and balance problems (12 patients) and fractures (8 patients). In the cohort $79.9 \%$ of women and $88.6 \%$ of men were right handed. Using the EWGSOP2 provided cut-off points for low hand grip strength, 39.58\% of the women and $42.86 \%$ of the men were classified to have probable sarcopenia. In both men and women, 56\% had either malnutrition or were at risk of malnutrition according the MNA- SF. The mean length of stay was 10 days. The median SARC-F score of 3 for women and 4 for men indicates a relatively high presence of self-reported characteristics of sarcopenia. A median score of 1.50 for women and 2 for men, on the FRAIL scale, indicates the presence of multiple characteristics of frailty.

\section{Correlation between lower arm muscle thickness and handgrip strength}

The mean handgrip strength was $16.75 \pm 4.82 \mathrm{~kg}$ for women and $28.60 \pm 9.32 \mathrm{~kg}$ for men. The mean lower arm muscle thickness was $37.28 \pm 5.91 \mathrm{~mm}$ for women and $41.61 \pm 4.94 \mathrm{~mm}$ for men. The square root of the handgrip strength had to be taken to correct for skewness and ensure a normal distribution according the Shapiro-Wilk test. The scatterplot and fit line for lower arm muscle thickness and the square root of the handgrip strength suggested a linear relationship between the two variables 


\begin{tabular}{|c|c|c|}
\hline & Women & Men \\
\hline Gender-n (\%) & $48(57.8)$ & $35(42.2)$ \\
\hline Age-years & $84.51 \pm 5.29$ & $84.19 \pm 6.01$ \\
\hline CIRS-G score-median (IOR) & $10(6)$ & $13(8)$ \\
\hline \multicolumn{3}{|l|}{ Alcohol-n (\%) } \\
\hline Total abstinence & $23(48.9)$ & $11(31.4)$ \\
\hline Weekly & $24(51.1)$ & $21(60.0)$ \\
\hline Daily & $0(0)$ & $3(8.6)$ \\
\hline \multicolumn{3}{|l|}{ Smoking-n (\%) } \\
\hline Non-smoker & $31(64.4)$ & $16(45.7)$ \\
\hline Ex-smoker & $15(31.3)$ & $18(51.4)$ \\
\hline Active smoker & $2(4.2)$ & $1(2.9)$ \\
\hline \multicolumn{3}{|l|}{ Reason for admission-n (\%) } \\
\hline Respiratory infection & $4(8.3)$ & $3(8.6)$ \\
\hline Urinary tract infection & 0 & $3(8.6)$ \\
\hline Other infections & $7(14.6)$ & $3(8.6)$ \\
\hline Gait and balance problem & $10(20.8)$ & $2(5.7)$ \\
\hline Hip fracture & $1(2.1)$ & $2(5.7)$ \\
\hline Other fracture & $4(8.3)$ & $1(2.9)$ \\
\hline Neoplasm related & $4(8.3)$ & $1(2.9)$ \\
\hline Gastrointestinal bleeding & $2(4.2)$ & $1(2.9)$ \\
\hline Other gastrointestinal disease & $3(6.3)$ & $4(11.4)$ \\
\hline $\begin{array}{l}\text { Transcatheter aortic valve } \\
\text { implantation }\end{array}$ & $2(4.2)$ & $6(17.1)$ \\
\hline Other cardiovascular disease & $2(4.2)$ & $3(8.6)$ \\
\hline Adverse drug event & $1(2.1)$ & $3(8.6)$ \\
\hline Pain problem & $1(2.1)$ & $2(5.7)$ \\
\hline Other & 7 (14.6) & $3(8.6)$ \\
\hline Handedness-right/left $\%$ & $97.9 / 2.1$ & $88.6 / 11.4$ \\
\hline Weight-kg & $63.06 \pm 14.50$ & $75.79 \pm 11.91$ \\
\hline Length-cm & $159.34 \pm 7.88$ & $174.06 \pm 6.40$ \\
\hline Body mass index $-\mathrm{kg} / \mathrm{m}^{2}$ & $24.80 \pm 5.28$ & $24.95 \pm 3.22$ \\
\hline Length lower arm-mm & $253.27 \pm 14.66$ & $274.72 \pm 15.29$ \\
\hline Muscle thickness-mm & $37.28 \pm 5.91$ & $41.61 \pm 4.94$ \\
\hline $\begin{array}{l}\text { Handgrip strength dominant } \\
\text { hand-kg }\end{array}$ & $16.75 \pm 4.82$ & $28.60 \pm 9.32$ \\
\hline $\begin{array}{l}\text { Presence sarcopenia } \\
\text { (EWGSOP2-criteria) } \\
\text { No sarcopenia-n (\%) } \\
\text { Probable sarcopenia-n (\%) }\end{array}$ & $\begin{array}{l}29(60.42) \\
19(39.58)\end{array}$ & $\begin{array}{l}20(57.14) \\
15(42.86)\end{array}$ \\
\hline Albumin-g/dL & $35.90 \pm 4.04$ & $36.59 \pm 5.07$ \\
\hline Prealbumin-mg/dL & $0.192 \pm 0.051$ & $0.213 \pm 0.057$ \\
\hline SARC-F score-median (IOR) & $3(4)$ & $4(5)$ \\
\hline FRAIL scale-median (IOR) & $1.5(1)$ & $2(2)$ \\
\hline MNA-SF-median (IOR) & $11(5)$ & $10(4)$ \\
\hline $\begin{array}{l}\text { Malnutrition (score } 0-7 \text { ) - n } \\
(\%)\end{array}$ & $10(20.8)$ & $3(8.8)$ \\
\hline
\end{tabular}

\begin{tabular}{|c|c|c|}
\hline & Women & Men \\
\hline $\begin{array}{l}\text { Risk of malnutrition (score } \\
8-11) \text { - n (\%) }\end{array}$ & $17(35.4)$ & $16(47.0)$ \\
\hline $\begin{array}{l}\text { Normal nutritional status } \\
\text { (score } 12-14)-n(\%)\end{array}$ & $21(43.9)$ & $15(44.1)$ \\
\hline Length of stay-days & $10.88 \pm 6.71$ & $10.11 \pm 8.12$ \\
\hline Medication on admission- $n$ & $6.77 \pm 3.79$ & $7.29 \pm 3.38$ \\
\hline \multicolumn{3}{|c|}{$\begin{array}{l}\text { Data shown as mean } \pm \text { standard deviation unless otherwise indicated. } \\
\text { IQR: Interquartile range, MNA-SF: Mini nutritional assessment-short form, EWGSOP2: } \\
\text { European Working Group on Sarcopenia in Older People }\end{array}$} \\
\hline
\end{tabular}

(Figure 3,4). The calculated Pearson's correlation coefficient between the square root of handgrip strength and lower arm muscle thickness was $0.287(p=0.051)$ for women and 0.361 $(p=0.036)$ for men. To further explore the relationship between handgrip strength and muscle thickness, an additional linear regression analysis was performed. Hereby only the data of the participating men was used since only the men's calculated Pearson's correlation coefficient $(0.361, p=0.036)$ was significant. Regression analysis showed that handgrip strength in men can be estimated using the following formula:

handgrip strength $(\mathrm{kg})=[2.773+0.061 \times$ lower arm muscle thickness $\left.\left(\mathrm{mm}^{2}\right)\right]$.

The adjusted $\mathrm{R}$ square of the linear regression analysis was 0.103. An additional bivariate correlation analysis (including: age, CIRS-G, weight, length,) was performed using a correlation matrix. No additional variables significantly correlated (Spearman's rho, $p>0.05$ ) to handgrip strength were found (Table 2).

\section{Discussion}

Using US-based muscle measurements, the SARCUS3 pilot study found a positive linear relationship between lower arm muscle quantity and handgrip strength in hospitalized older adults. The results were significant for men and borderline non-significant for women. Handgrip strength in men could be estimated using the following formula:

handgrip strength $(\mathrm{kg})=[2.773+0.061 \times$ lower arm muscle thickness $\left.\left(\mathrm{mm}^{2}\right)\right]$

Although there is some data on the relationship between lower arm muscle quantity and handgrip strength in healthy community dwelling adults $(22,33,34,43)$, this study provides for the first-time confirmation of this relationship in hospitalized older adults with serious co- morbidities.

There are multiple reasons however why the correlation was not as pronounced as one might expect. First of all, as noted by previous auteurs, there is an age-related decrease in the correlation between lower arm muscle thickness and handgrip strength $(22,33,34,43)$. As the population in our study [mean 
age in years: women $84.51 \pm 5.29$, men $84.19 \pm 6.01$ ) was significant older than in previous studies (mean age in years for women and men: $23 \pm 3,24 \pm 4(43) ; 73 \pm 3,74 \pm 3$ (34); women/men together $31 \pm 14$ (22); and women/men together aged 20 to 89 (33)] it was expected that the correlation

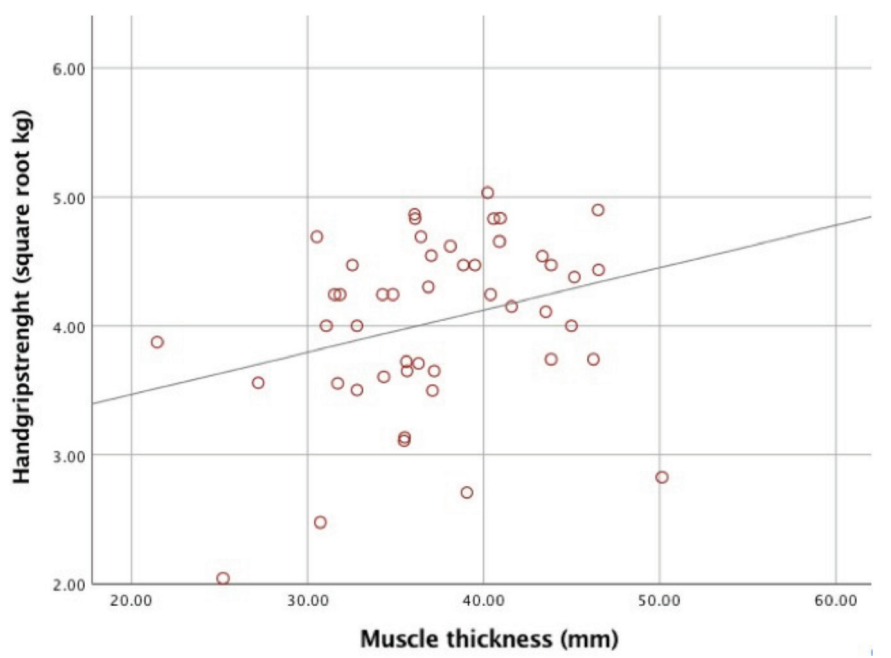

Figure 3. Scatterplot of the muscle thickness and the square root of handgrip strength (women). The square root of the handgrip strength had to be taken to correct for skewness and ensure a normal distribution according the Shapiro-Wilk test

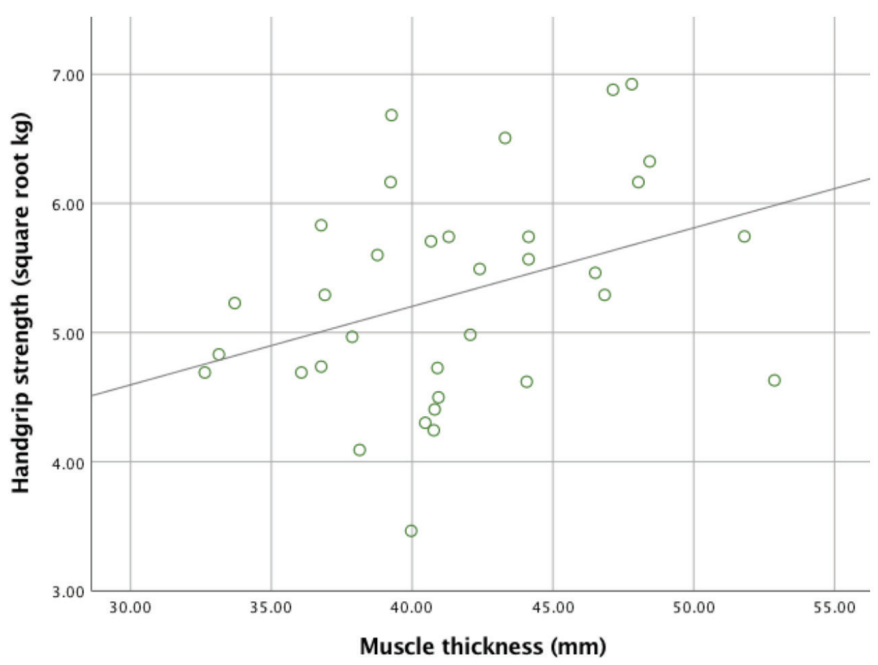

Figure 4. Scatterplot of the muscle thickness and the square root of handgrip strength (men). The square root of the handgrip strength had to be taken to correct for skewness and ensure a normal distribution according the Shapiro-Wilk test

\begin{tabular}{|l|l|l|}
\hline \multicolumn{3}{|l|}{ Table 2. Bivariate correlations } \\
\hline Independent variable & Women & Men \\
\hline Age & $-0.105(0.478)$ & $-0.336(0.52)$ \\
\hline CIRS-G & $-0.110(0.456)$ & $-0.163(0.356)$ \\
\hline Length & $0.120(0.421)$ & $0.209(0.237)$ \\
\hline Weight & $0.256(0.083)$ & $0.331(0.056)$ \\
\hline
\end{tabular}

Data shown as Spearman's rho correlation coefficient ( $p$-value) between independent variable and handgrip strength between lower arm muscle mass and handgrip strength would be lower as well.

Another reason for the weak relationship observed between lower arm muscle quantity and handgrip strength is certainly the absence of clear qualitative muscle measurements (44-46). Qualitative determinants like age-related adipose or connective tissue infiltration of the muscle, changes in myofiber size, changes in muscle metabolism, a reduced capillary density or neural changes that have been described in previous studies might be equally important as a decline in muscle quantity, certainly in an older age cohort (47). The calculated linear regression analysis in men showed an adjusted $\mathrm{R}$ square of 0.103 . This means that only $10.3 \%$ of the witnessed variability in handgrip strength can be explained by the muscle thickness.

This showing a certain margin of error for the current formula [handgrip strength $(\mathrm{kg})=[2.773+0.061 \times$ lower arm muscle thickness $\left.(\mathrm{mm})^{2}\right]$. The absence of a significant correlation in the additional bivariate analysis suggests other variables, for example muscle quality, must therefore contribute to the witnessed variability in handgrip strength.

Previous studies have shown an age-related decline in handgrip strength (7). Our results on handgrip strength (mean handgrip strength $16.75 \pm 4.82 \mathrm{~kg}$ for women and $28.60 \pm 9.32 \mathrm{~kg}$ for men) are, both for men and woman, within the $25-50^{\text {th }}$ percentile of the age and gender specified reference range (7). As expected a significant portion of the patients was diagnosed with probable sarcopenia according the EWGSOP2 criteria (39.58\% women, $42.86 \%$ men) on account of having low handgrip strength (6). The absence of a significant correlation between age and handgrip strength in the bivariate analysis is probably due to the small sample size and limited age-range of the study population.

The observed relationship between lower arm muscle thickness and handgrip strength was lower in our study than in previous US-based observations regarding lower limb muscle $(48,49)$. Prior studies reported on the relationship between muscle thickness of the quadriceps and muscle strength (correlation coefficient: $0.422, p<0.001)(48)$ and the thickness of the musculus rectus femoris and maximum isometric voluntary contraction (Pearson correlation coefficients: $0.834, p<0.001)(49)$. A study investigating age-related site-specific muscle wasting showed an age-related increased muscle loss in the upper leg muscles (50). However, the age-related effect on lower arm muscles was rather small. The absence of significant age- related lower arm muscle loss combined with an age-related decline in handgrip strength further emphasizes the importance of muscle quality as a predictor of lower arm muscle strength in later life.

Although this was not the primary aim of our study, the SARCUS3 study showed that US- based measurements of the lower arm 
muscles in older adults are feasible to obtain in regular clinical practice and could therefore be useful for the screening of low muscle mass in this cohort. In contrast to previous studies, a seated patient position was chosen with a flexed arm (elbow $120^{\circ}$ ) on the table instead of a standing position with stretched arms $(22,33,34,43)$. This new approach is more appropriate for studies in older adults for several reasons. First of all, a seated position is physical less demanding for older adults than a standing position. Secondly, a rested flexed arm position is a more natural body posture in contrast to a stretched arm, making it easier for patients to maintain the same posture for the duration of the examination. In a standing position, test subjects always need to contract muscles to maintain the "ideal" test position. This is not the case in a seated position, leading to less variation in measurements. Finally, the table surface can be used to rest the probe, reducing the risk of vertical probe displacement when taking repetitive measurements.

Similar to previous studies the choice was made to measure an entire muscle compartment rather than a single muscle $(22,33,34,43)$. Although this increases the risk of including nonmuscle tissue as part of the measurement, this is a more feasible approach for the screening of sarcopenia in older adults. As a consequence of the difference in our study protocol to previous studies, a direct comparison between the measured muscle thickness was not possible $(22,33,34,43)$.

The SARCUS 3 study had several strengths. All measurements were acquired using a strict protocol, with special attention for standardization. All data and measurements were obtained by one trained researcher, excluding any inter-observer disagreement.

\section{Study Limitations}

The SARCUS3 study has some limitations. First of all, the reason for admission could have influenced patient's handgrip strength and lower arm muscle thickness. We tried to minimize this by excluding severely ill patients who were not able to comply with the study protocol. Furthermore, we believe that the impact of acute muscle wasting would have been limited.

Experimental research showed that older adults have a total lean mass decreased of $\pm 4 \%$ after 5 days of total bed rest (51). Patients where however not allowed to be bedridden in order to comply with the study protocol, additionally early mobilization is regarded as usual care on our geriatric ward. Secondly, we excluded patients with cognitive problems to ensure correct data collection and adherence to the study protocol. Thus, making it unsure if our findings apply to all hospitalized older adults. Lastly, a direct comparison between US and CT, MRI, CT or BIA based measurement would have given additional insight on potential US based muscle measurement of variables like pennation angle (the angle formed between fiber insertions and the aponeurosis in penniform muscles), fascicle length or echo intensity $(15,18)$. This was however beyond the scope of this pilot study, primarily because the focus was placed on feasibility in clinical practice.

\section{Conclusion}

The results of this study show for the first time a significant positive relationship for men and a borderline significant relationship for women between lower arm muscle thickness and handgrip strength in hospitalized older adults. Up to $10.3 \%$ of the measured variability of the handgrip strength in men can be explained by muscle thickness alone. Further research is necessary to look into other relevant factors.

\section{Ethics}

Ethics Committee Approval: This study was approved by our Local Hospital's Ethics Committee (approval no: 5226).

Informed Consent: Written informed consent was obtained from all subjects.

Peer-review: Externally peer-reviewed.

\section{Authorship Contributions}

Surgical and Medical Practices: T.A., A.M.D.C., V.M., S.P., Concept: T.A., A.M.D.C., V.M., S.P., Design: T.A., A.M.D.C., V.M., S.P., Data Collection or Processing: T.A., A.M.D.C., S.P., Analysis or Interpretation: T.A., A.M.D.C., S.P., Literature Search: T.A., A.M.D.C., S.P., Writing: T.A., A.M.D.C., S.P.

Conflict of Interest: No conflict of interest was declared by the authors.

Financial Disclosure: The authors declared that this study received no financial support.

\section{References}

1. Rosenberg IH. Summary comments. American Journal of Clinical Nutrition 1989;50:1231-1233.

2. Bijlsma AY, Meskers CG, Westendorp RG, Maier AB. Chronology of agerelated disease definitions: osteoporosis and sarcopenia. Ageing Res Rev 2012;11:320-324.

3. Beaudart C, Zaaria M, Pasleau F, Reginster JY, Bruyère O. Health Outcomes of Sarcopenia: A Systematic Review and Meta-Analysis. PLoS One 2017;12:e0169548.

4. Tsekoura M, Kastrinis A, Katsoulaki M, Billis E, Gliatis J. Sarcopenia and Its Impact on Quality of Life. Adv Exp Med Biol 2017;987:213-218.

5. Cruz-Jentoft AJ, Baeyens JP, Bauer JM, Boirie Y, Cederholm T, Landi F, Martin FC, Michel JP, Rolland Y, Schneider SM, Topinková E, Vandewoude M, Zamboni M; European Working Group on Sarcopenia in Older People. Sarcopenia: European consensus on definition and diagnosis: Report of the European Working Group on Sarcopenia in Older People. Age Ageing 2010;39:412-423.

6. Cruz-Jentoft $A J$, Baha $t G$, Bauer J, Boirie $Y$, Bruyère 0 , Cederholm $T$, et al.Sarcopenia: revised European consensus on definition and diagnosis. Age and ageing 2019;48:16-31. 
7. Dodds RM, Syddall HE, Cooper R, Benzeval M, Deary IJ, Dennison EM, Der G, Gale CR, Inskip HM, Jagger C, Kirkwood TB, Lawlor DA, Robinson SM, Starr JM, Steptoe A, Tilling K, Kuh D, Cooper C, Sayer AA. Grip strength across the life course: normative data from twelve British studies. PLoS One 2014;9:e113637.

8. Rijk JM, Roos PR, Deckx L, van den Akker M, Buntinx F. Prognostic value of handgrip strength in people aged 60 years and older: A systematic review and meta-analysis. Geriatr Gerontol Int 2016;16:5-20.

9. Hiraoka $A$, Tamura $R, O$ ka $M$, Izumoto $H$, Ueki $H$, Tsuruta $M$, Yoshino $T$, Ono A, Aibiki T, Okudaira T, Yamago $H$, Suga $Y$, Iwasaki $R$, Mori $K$, Miyata $H$, Tsubouchi E, Kishida M, Ninomiya T, Hirooka M, Abe M, Matsuura B, Hiasa $Y$, Kino T, Yamamoto $K$, Michitaka K. Prediction of risk of falls based on handgrip strength in chronic liver disease patients living independently. Hepatol Res 2019;49:823-829.

10. Kunutsor SK, Seidu S, Voutilainen A, Blom AW, Laukkanen JA. Handgrip strength- ariskindicator for future fractures in the general population:findings from a prospective study and meta-analysis of 19 prospective cohort studies. GeroScience 20202020/08/19.

11. Wiśniowska-Szurlej A, Ćwirlej-Sozańska A, Wołoszyn N, Sozański B, Wilmowska-Pietruszyńska A. Association between Handgrip Strength, Mobility, Leg Strength, Flexibility, and Postural Balance in Older Adults under Long-Term Care Facilities. Biomed Res Int 2019;2019:1042834.

12. Rolland $Y$, Lauwers-Cances $V$, Cesari $M$, Vellas $B$, Pahor $M$, Grandjean $H$. Physical performance measures as predictors of mortality in a cohort of community-dwelling older French women. Eur J Epidemiol 2006;21:113122.

13. De Buyser SL, Petrovic M, Taes YE, Toye KR, Kaufman JM, Goemaere S. Physical function measurements predict mortality in ambulatory older men. Eur J Clin Invest 2013;43:379-386.

14. Gonzalez MC, Barbosa-Silva TG, Heymsfield SB. Bioelectrical impedance analysis in the assessment of sarcopenia. Curr Opin Clin Nutr Metab Care 2018;21:366-374.

15. Ticinesi A, Meschi T, Narici MV, Lauretani F, Maggio M. Muscle Ultrasound and Sarcopenia in Older Individuals: A Clinical Perspective. J Am Med Dir Assoc 2017;18:290-300.

16. Gould H, Brennan SL, Kotowicz MA, Nicholson GC, Pasco JA. Total and appendicular lean mass reference ranges for Australian men and women: the Geelong osteoporosis study. Calcif Tissue Int 2014;94:363-372.

17. Studenski SA, Peters KW, Alley DE, Cawthon PM, McLean RR, Harris TB, Ferrucci L, Guralnik JM, Fragala MS, Kenny AM, Kiel DP, Kritchevsky SB, Shardell MD, Dam $\Pi$, Vassileva MT. The FNIH sarcopenia project: rationale, study description, conference recommendations, and final estimates. J Gerontol A Biol Sci Med Sci 2014;69:547-558.

18. Perkisas $S$, Bastijns $S$, Baudry $S$, Bauer J, Beaudart C, Beckwée D, CruzJentoft $A$, Gasowski J, Hobbelen $H$, Jager-Wittenaar $H$, Kasiukiewicz $A$, Landi $F$, Małek $M$, Marco $E$, Martone AM, de Miguel AM, Piotrowicz $K$, Sanchez E, Sanchez-Rodriguez D, Scafoglieri A, Vandewoude M, Verhoeven V, Wojszel ZB, De Cock AM. Application of ultrasound for muscle assessment in sarcopenia: 2020 SARCUS update. Eur Geriatr Med 2021;12:45-59.

19. Sanada K, Kearns CF, Midorikawa T, Abe T. Prediction and validation of total and regional skeletal muscle mass by ultrasound in Japanese adults. Eur J Appl Physiol 2006;96:24-31.

20. Thomaes T, Thomis M, Onkelinx S, Coudyzer W, Cornelissen V, Vanhees L. Reliability and validity of the ultrasound technique to measure the rectus femoris muscle diameter in older CAD-patients. BMC Med Imaging 2012;12:7.

21. Reeves ND, Maganaris CN, Narici MV. Ultrasonographic assessment of human skeletal muscle size. Eur J Appl Physiol 2004;91:116-118.

22. Abe T, Nakatani M, Loenneke JP. Relationship between ultrasound muscle thickness and MRI-measured muscle cross-sectional area in the forearm: a pilot study. Clin Physiol Funct Imaging 2018;38:652-655.
23. Nijholt W, Scafoglieri A, Jager-Wittenaar H, Hobbelen JSM, van der Schans $\mathrm{CP}$. The reliability and validity of ultrasound to quantify muscles in older adults: a systematic review. J Cachexia Sarcopenia Muscle 2017;8:702-712.

24. Tillquist M, Kutsogiannis DJ, Wischmeyer PE, Kummerlen C, Leung R, Stollery D, Karvellas CJ, Preiser JC, Bird N, Kozar R, Heyland DK. Bedside ultrasound is a practical and reliable measurement tool for assessing quadriceps muscle layer thickness. JPEN J Parenter Enteral Nutr 2014;38:886-890.

25. Wallwork TL, Hides JA, Stanton WR. Intrarater and interrater reliability of assessment of lumbar multifidus muscle thickness using rehabilitative ultrasound imaging. J Orthop Sports Phys Ther 2007;37:608-612.

26. Cuellar WA, Blizzard L, Callisaya ML, Hides JA, Jones G, Ding C, Winzenberg TM. Test-retest reliability of measurements of abdominal and multifidus muscles using ultrasound imaging in adults aged 50-79 years. Musculoskelet Sci Pract 2017;28:79-84.

27. Latey PJ, Burns J, Nightingale EJ, Clarke JL, Hiller CE. Reliability and correlates of cross-sectional area of abductor hallucis and the medial belly of the flexor hallucis brevis measured by ultrasound. J Foot Ankle Res 2018;11:28.

28. Crofts G, Angin S, Mickle KJ, Hill S, Nester CJ. Reliability of ultrasound for measurement of selected foot structures. Gait Posture 2014;39:35-39.

29. Mohseny B, Nijhuis TH, Hundepool CA, Janssen WG, Selles RW, Coert JH. Ultrasonographic quantification of intrinsic hand muscle cross-sectional area; reliability and validity for predicting muscle strength. Arch Phys Med Rehabil 2015;96:845-853.

30. Ismail C, Zabal J, Hernandez HJ, Woletz P, Manning H, Teixeira C, DiPietro L, Blackman MR, Harris-Love MO. Diagnostic ultrasound estimates of muscle mass and muscle quality discriminate between women with and without sarcopenia. Front Physiol 2015;6:302.

31. Kellis E, Galanis N, Natsis K, Kapetanos G. Validity of architectural properties of the hamstring muscles: correlation of ultrasound findings with cadaveric dissection. J Biomech 2009;42:2549-2454.

32. Tosovic D, Muirhead JC, Brown JM, Woodley SJ. Anatomy of the long head of biceps femoris: An ultrasound study. Clin Anat 2016;29:738-745.

33. Abe T, Thiebaud RS, Loenneke JP. Age-related change in handgrip strength in men and women: is muscle quality a contributing factor? Age (Dordr) 2016;38:28.

34. Abe T, Thiebaud RS, Loenneke JP, Ogawa M, Mitsukawa N. Association between forearm muscle thickness and age-related loss of skeletal muscle mass, handgrip and knee extension strength and walking performance in old men and women: a pilot study. Ultrasound Med Biol 2014;40:20692075.

35. Miller MD, Paradis CF, Houck PR, Mazumdar S, Stack JA, Rifai AH, Mulsant $B$, Reynolds CF 3rd. Rating chronic medical illness burden in geropsychiatric practice and research: application of the Cumulative IIIness Rating Scale. Psychiatry Res 1992;41:237-248.

36. Rubenstein LZ, Harker JO, Salvà A, Guigoz Y, Vellas B. Screening for undernutrition in geriatric practice: developing the short-form mininutritional assessment (MNA-SF). J Gerontol A Biol Sci Med Sci 2001;56:M366-372.

37. Malmstrom TK, Miller DK, Simonsick EM, Ferrucci L, Morley JE. SARC-F: a symptom score to predict persons with sarcopenia at risk for poor functional outcomes. J Cachexia Sarcopenia Muscle 2016;7:28-36.

38. Abellan van Kan G, Rolland Y, Bergman H, Morley JE, Kritchevsky SB, Vellas B. The I.A.N.A Task Force on frailty assessment of older people in clinical practice. J Nutr Health Aging 2008;12:29-37.

39. Morley JE, Malmstrom TK, Miller DK. A simple frailty questionnaire (FRAIL) predicts outcomes in middle aged African Americans. J Nutr Health Aging 2012;16:601-608.

40. Welch C, K Hassan-Smith Z, A Greig C, M Lord J, A Jackson T. Acute Sarcopenia Secondary to Hospitalisation - An Emerging Condition Affecting Older Adults. Aging Dis 2018;9:151-164. 
41. Roberts HC, Denison HJ, Martin HJ, Patel HP, Syddall H, Cooper C, Sayer AA. A review of the measurement of grip strength in clinical and epidemiological studies: towards a standardised approach. Age Ageing 2011;40:423-429.

42. Sousa-Santos AR, Amaral TF. Differences in handgrip strength protocols to identify sarcopenia and frailty - a systematic review. BMC Geriatr 2017; 17:238.

43. Abe $T$, Counts BR, Barnett BE, Dankel SJ, Lee K, Loenneke JP. Associations between Handgrip Strength and Ultrasound-Measured Muscle Thickness of the Hand and Forearm in Young Men and Women. Ultrasound Med Biol 2015;41:2125-2130.

44. Visser M, Newman AB, Nevitt MC, Kritchevsky SB, Stamm EB, Goodpaster $\mathrm{BH}$, Harris TB. Reexamining the sarcopenia hypothesis. Muscle mass versus muscle strength. Health, Aging, and Body Composition Study Research Group. Ann N Y Acad Sci 2000;904:456-461.

45. Fragala MS, Kenny AM, Kuchel GA. Muscle quality in aging: a multidimensional approach to muscle functioning with applications for treatment. Sports Med 2015;45:641-658.

46. Clark BC, Manini TM. Functional consequences of sarcopenia and dynapenia in the elderly. Curr Opin Clin Nutr Metab Care 2010;13:271-276.
47. Distefano G, Goodpaster BH. Effects of Exercise and Aging on Skeletal Muscle. Cold Spring Harb Perspect Med 2018;8:a029785.

48. Akazawa N, Harada K, Okawa N, Tamura K, Moriyama H. Muscle mass and intramuscular fat of the quadriceps are related to muscle strength in nonambulatory chronic stroke survivors: A cross-sectional study. PLoS One 2018;13:e0201789.

49. Strasser EM, Draskovits $T$, Praschak M, Quittan M, Graf A. Association between ultrasound measurements of muscle thickness, pennation angle, echogenicity and skeletal muscle strength in the elderly. Age (Dordr) 2013;35:2377-2388.

50. Abe T, Loenneke JP, Thiebaud RS, Fukunaga T. Age-related site-specific muscle wasting of upper and lower extremities and trunk in Japanese men and women. Age (Dordr) 2014;36:813-821.

51. Tanner RE, Brunker LB, Agergaard J, Barrows KM, Briggs RA, Kwon OS, Young LM, Hopkins PN, Volpi E, Marcus RL, LaStayo PC, Drummond MJ. Age-related differences in lean mass, protein synthesis and skeletal muscle markers of proteolysis after bed rest and exercise rehabilitation. J Physiol 2015;593:4259-4273.

\section{Supplementary Material}

\section{Exclusion criteria}

All patients with a history of/or the presence of a paresis/paralysis of the dominant upper limb due to a stroke will be excluded. All patient with severe electrolyte disturbances at admission will be excluded because of the potential effect on muscle contraction. Patients who are clinically either hyper- or hypovolemic will be excluded because fluid shifts could influence the US measurement results. All patients who are unable to comply with the given instructions about limb positioning will be excluded. Patients with a history of systemic connective tissue disorders, myositis, calcification and ossification of muscle, systemic atrophies primarily affecting the central nervous system and demyelinating diseases of the central nervous system will be excluded because of the uncertain effect muscle quantity and function. All patient with partial or total parenteral and/or intravenous nutrition will be excluded. 